\title{
Application of Hybrid Particle Swarm Algorithm for Water Supply Optimization Scheduling in Mountain Scenic Spot
}

\author{
Jie $\mathrm{LI}^{1, \mathrm{a}^{*}}$, Yi HUANG ${ }^{1, \mathrm{~b}}$ and Qiang $\mathrm{LI}^{1, \mathrm{c}}$, \\ Shan-shan $\mathrm{ZHU}^{1, d}$, Liang LING ${ }^{2}$, Cheng FANG ${ }^{2}$ \\ ${ }^{1}$ School of Computer and Information Hefei University of Technology, Anhui, 230009, P.R.China \\ ${ }^{2}$ Mount Huangshan Scenic Area Water Supply Ltd. Co. Anhui, 242700, P.R.China) \\ a lijie_62@163.com, ${ }^{\mathrm{b}}$ yihuang63@163.com, ${ }^{\mathrm{d}}$ zhushanshan7s601@163.com \\ * Jie LI (1962- ), male, associate professor, major research: computer applications \\ technology,computer control
}

\begin{abstract}
Keywords: particle swarm algorithm; penalty method; non-linear constrain; optimization scheduling; objective function; optimal solution

Abstract: As the characteristics of mountain scenic spot possess complicated topography, great elevation differences, high water diversion costs, limited reservoir storage capacity, uneven distribution of rainfall, coupled with hysteresis nature of the manual diversion, scarcity of water resources has become a major factor restricting the development of scenic spots. Therefore, it is an effective way to solve the water shortage problem through optimizing the existing water supply scheduling scheme. Water supply optimization scheduling in mountain scenic spot is a typical non-linear, in-differentiable, constraint problem. This paper aims at the problem of water supply optimization scheduling in mountain scenic spot and introduces a method to solve the hybrid particle swarm algorithm of nonlinear constrained optimization. The algorithm combines the advantages of penalty method and particle swarm optimization algorithms, employs particle swarm algorithm to solve complex objective function, and utilizes penalty method to introduce evaluation mechanism of penalty factor to deal with the constraint problem. In the end, this will be applied in water supply optimization scheduling of Huangshan Scenic Area. Experimental results demonstrate that the algorithm shows good performance in solving the water supply scheduling problem of mountain scenic spot.
\end{abstract}

\section{Introduction}

Mountain scenic area is very different from urban water supply in the rainfall, water resources scheduling and storage mode. Cities generally have relatively ample supply of water and achieve the purpose of optimizing the layout through rational optimization of the water supply network. However, it lacks large-scale mountain-resort natural water sources, so the rational allocation of the existing water resources is the key to solve the water shortage problem.

Take Huangshan Scenic Area as an example, the resort possesses complex climate, limited water resources. Its total capacity is 431,800 cubic meters with available and effective storage capacity of 391,800 cubic meters, which mainly comes from rainfall. Huangshan belongs to the mountain terrain that is complex and owns a large gap. Due to the subtropical monsoon climate and specific geographical conditions, this area has uneven rainfall distribution.

Annual late spring and early summer, the resort owns rich rainfall. While after the rainy season, it appears late summer, autumn and winter drought year after year and water is much scarce during the dry season. Water is very precious there. At the same time, water resources in the mountain resort scheduling will consume enormous power. Therefore, it is important to realize the rational management of water resources.

Typical optimization algorithms such as particle swarm optimization, genetic algorithm show better performance in the unconstrained nonlinear problems, but easy to fall into local optima when dealing with constraints. In this paper, combine particle swarm algorithm with penalty number, 
optimizing water supply scheduling in mountain-resort. Take Huangshan water supply system as the study case, compare optimization results with genetic algorithm.

\section{Model Introduction}

PSO (Particle Swarm Optimization, PSO) algorithm is an optimization algorithm proposed by James Kennedy and Russell Eberhart in 1995. In the PSO algorithm, optimized solution for each issue is a bird in the searching space, to be abstract as particles with no quality and volume, and extending it to the $\mathrm{N}$-dimensional space.

Position of the particle in the $\mathrm{N}$-dimensional space is represented by a vector, each particle speed is also represented as a vector. All particles have a fitness value determined by optimization function, each particle has a speed determining the direction and distance they fly. Particle know the best position they find so far, it can be seen as a particle flying experience, speed and position of a particle updated in accordance with the following formula:

$$
\begin{aligned}
& v_{i, n}(t+1)=w v_{i, j}(t)+c_{1} r_{1}\left[p_{i, j}-x_{i, j}(t)\right]+ \\
& c_{2} r_{2}\left[p_{g, j}-x_{i, j}(t)\right] \\
& x_{i, j}(t+1)=x_{i, j}(t)+v_{i, j}(t+1), j=1,2, \ldots, d .
\end{aligned}
$$

Among them, $\mathrm{w}$ is the inertia factor, ${ }^{c_{1}}$ and ${ }^{c}{ }_{2}$ are positive learning factor, ${ }_{1}$ and $r_{2}$ are random number uniformly distributed between 0-1. A particle through its own experience and the experience of the best companions determines the next movement, until it finds a global or local optimal solution.

Through the principle of particle swarm algorithm we can find that PSO unlike genetic algorithm that is crossover and mutation reducing the complexity of the algorithm, it can show good performance in the nonlinear non-binding question, but there are some limitations on the constraints problem solving.

Constrained optimization problems can generally be described as follows:

$$
\min f(x)=\text { s.t. }\left\{\begin{array}{l}
g_{j}(x) \leq 0, j=1,2, \ldots \ldots, j \\
h_{k}(x)=0, k=1,2, \ldots \ldots k \\
x_{i}^{m}<x_{i}<x_{i}^{n}, i=1,2, \ldots \ldots d
\end{array} .\right.
$$

Among them, $x=\left(x_{1}, x_{2, \ldots . . .} x_{d}\right)$ is the dimensional decision vector, which is the unknown number of the problem to be solved, $f(x)$ is the objective function, $\mathrm{g}_{j}(x)$ is the $\mathrm{j}$-th inequality constraint, $h_{k}(x)$ is the $\mathrm{k}$-th inequality constraints, ranging from vector $\mathrm{x}$ of $\left[x_{i}^{m}, x_{i}^{n}\right]$. Constrained optimization problem is ensuring $\mathrm{x}$ satisfies the above equation, the premise of inequality constraints within the definition of the objective function of variables to find optimal solutions.

If $\mathrm{x}$ is contrary to at least one constraint, it becomes infeasible; if $\mathrm{x}$ satisfies all the constraints, claimed it as a viable solution, the set of all feasible solutions become feasible region. Ultimate goal of Constrained Optimization is to find the best solution in $\mathrm{x}^{*}$ feasible region, making $f\left(x^{*}\right)$ to be the minimized.

\section{PSO Algorithm based on Penalty Number}

Penalty number can handle general constrained optimization problem, and its basic idea is to change constrained optimization problem into unconstrained problem to solve. Penalty function 
when constructed makes infeasible point value equal to an infinite value, Penalty value at feasible point values are the same with the original target values.

Based on the thinking of the penalty number, this paper will combine the standard PSO algorithm and penalty numbers inside and outside typical point method for solving nonlinear constrained optimization in mountain scenic spot of scheduling multiple-reservoirs. The objective function is introduced to penalty number. The construction augmented objective function is:

$$
F(x)=f(x)+\lambda_{k} \sum_{i=1}^{m} \frac{1}{g_{i}(x)}+\frac{1}{\sqrt{\lambda_{k}}} \sum_{j=1}^{n}\left[h_{j}(x)\right]^{2}
$$

Where $F(x)$ is augmented objective function, $f(x)$ is the original objective function, $g_{j}(x)$ is inequality constraints, $h_{j}(x)$ is the equality constraints, $\lambda_{k}$ is the penalty factor. With the increasing of the number of iterations, augmented objective function and objective function value difference are closer to zero. In this case, constrained optimization problem is transformed into unconstrained optimization. The optimal solution of unconstrained objective function $F(X)$ is the constraint of the objective function $f(x)$ optimal solution.

The basic idea: After the constraint problem changes into unconstrained optimization problems, iterative constantly adjust the penalty factor $\lambda_{1}, \lambda_{2}$ by iterating so that the sequence of new unconstrained objective function $F(x)$ minimum point gradually converge to the original constrained optimization objective function $f(x)$ the constraints optimal solution. For this reason, when constructing a complex punishment function, the following three properties must simultaneously exist, namely:

$$
\begin{aligned}
& \lim _{k \rightarrow \infty} \lambda_{1} \sum_{i=1}^{m} \frac{1}{g_{i}(x)}=0 . \\
& \lim _{k \rightarrow \infty} \lambda_{2} \sum_{j=1}^{n}\left[h_{j}(x)\right]^{2}=0 . \\
& \lim _{k \rightarrow \infty}|F(x)-f(x)|=0 .
\end{aligned}
$$

Here $\mathrm{k}$ refers to the number of iterations.

According to the above processing method, the use of PSO algorithm and penalty function combination based on PSO algorithm framework construct the evaluation function through punish method. The constrained optimization problem has been changed into unconstrained optimization. Evaluation function is constructed as follows:

$$
F(x)=f(x)+\lambda(t) H(x), x \in S \subset R^{d} .
$$

In this formula, $f(x)$ is the objective function of the constrained optimization, $\lambda(t)=\sqrt{t}$ 
is the penalty function factor, $t$ is the iteration number of particle swarm optimization. $H(x)$ is a penalty item, constructed as follows:

$$
\begin{aligned}
& H(X)=\sum_{m=1}^{n} \theta\left(p_{m}(x)\right) p_{m}(x)^{\alpha} \\
& p_{m}(x)=\left\{\begin{array}{l}
\max \left\{0, g_{m}(x)\right\}, \quad m=1,2, \ldots \ldots, \quad J \\
\left|h_{m}(x)\right|, \quad m=J+1, \quad J+2, \ldots \ldots, \quad n
\end{array}\right\} .
\end{aligned}
$$

In the above two formulas, $n$ is the number of constraints; $p_{m}(x)$ is a violation of the constraint function; $g_{m}(x)$ is the inequality constraint functions; $h_{m}(x)$ is the equality constraints function; $\theta\left(p_{m}(x)\right)$ is multistage distribution function; $\alpha$ is punitive series. Functions $p_{m}(x)$, $\theta\left(p_{m}(x)\right)$ and $\alpha$ are dependent on constrained optimization problems, its value rules:

(1) When $p_{m}(x)<1, \quad \alpha=1$

when $0<p_{m}(x) \leq 0.001, \theta\left(p_{m}(x)\right)=10$;

when $0.001<p_{m}(x) \leq 0.1, \theta\left(p_{m}(x)\right)=20$;

when $0.1<p_{m}(x)<1, \theta\left(p_{m}(x)\right)=100$.

(2) when $p_{m}(x) \geq 1, \alpha=2, \theta\left(p_{m}(x)\right)=300$.

Based on PSO algorithm framework, introduce into penalty function, the flowchart of the algorithm is as Fig. 1.

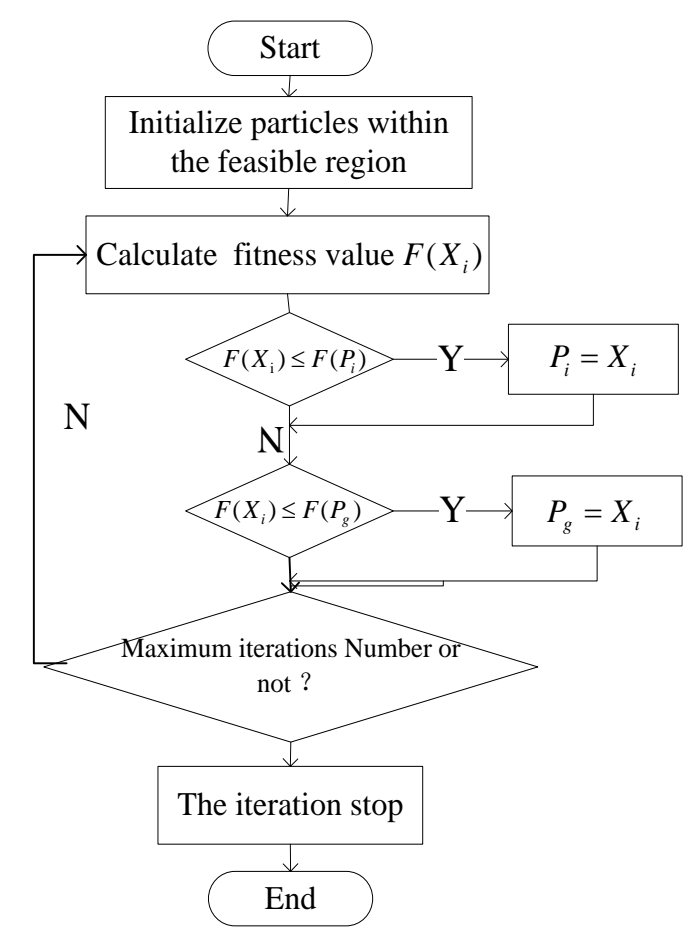

Fig. 1 Program flowchart of the Hybrid Particle Swarm Optimization 
Here are the experimental results that the PSO algorithm and penalty function combined to solve typical nonlinear constrained optimization problem. The paper has compared with other solution.

Test function 1: Known best solution for this function is $f\left(x^{*}\right)=0.25$.

$\min f(x)=100\left(x_{2}-x_{1}^{2}\right)^{2}+\left(1-x_{1}\right)^{2}$

s.t. $\left\{\begin{array}{l}g_{1}(x)=-x_{1}-x_{2}^{2} \leq 0 \\ g_{2}(x)=-x_{1}^{2}-x_{2} \leq 0 \\ -0.5 \leq x_{1} \leq 0.5, x_{2} \leq 1.0\end{array}\right\}$.

Test function 2: Known best solution for this function is $f\left(x^{*}\right)=13.59085$.

$\min f(x)=\left(x_{1}^{2}+x_{2}-11\right)^{2}+\left(x_{1}+x_{2}^{2}-7\right)^{2}$.

$s, t,\left\{\begin{array}{l}g_{1}(x)=\left(x_{1}-0.05\right)^{2}+\left(x_{2}-2.5\right)^{2}-4.84 \leq 0 \\ g_{2}(x)=4.84-x_{1}^{2}-\left(x_{2}-2.5\right)^{2} \leq 0 \\ 0 \leq x_{1} \leq 6,0 \leq x_{2} \leq 6\end{array}\right\}$.

Test function 3: Known best solution for this function is $f\left(x^{*}\right)=680.6300573$.

$$
\begin{aligned}
& \min f(x)=\left(x_{1}-10\right)^{2}+5\left(x_{2}-12\right)^{2}+x_{3}^{4}+3\left(x_{4}-11\right)^{2} . \\
& +10 x_{5}^{6}+7 x_{6}^{2}+x_{7}^{4}-4 x_{6} x_{7}-10 x_{6}-8 x_{7} \\
& \text { s.t. }=\left\{\begin{array}{l}
g_{1}(x)=-127+2 x_{1}^{2}+3 x_{2}^{4}+x_{3}+4 x_{2}^{4}+5 x_{5} \leq 0 \\
g_{2}(x)=-282+7 x_{1}+3 x_{2}+10 x_{3}^{2}+x_{4}-x_{5} \leq 0 \\
g_{3}(x)=-196+23 x_{1}+x_{2}^{3}+6 x_{6}^{2}-8 x_{7} \leq 0 \\
g_{4}(x)=4 x_{1}^{2}+x_{1}^{2}-3 x_{1} x_{2}+2 x_{3}^{2}+5 x_{6}-11 x_{7} \leq 0 \\
-10 \leq x_{j} \leq 10, i=1,2, \ldots \ldots, 7
\end{array}\right\} .
\end{aligned}
$$

Table 1 Result of test function 1

\begin{tabular}{clc}
\hline Algorithm & Best solution & Mean value \\
\hline \multirow{2}{*}{ Genetic Algorithm } & $0.25,0.26,0.26,0.25,0.27$, & \\
& $0.26,0.25,0.25,0.27,0.25$ & 0.257 \\
This paper & $0.25,0.25,0.26,0.25,0.25$, & \multirow{2}{*}{0.253} \\
& $0.26,0.25,0.25,0.26,0.25$ & \\
\hline
\end{tabular}


Table 2 Results of test function 2

\begin{tabular}{cclll}
\hline \multirow{2}{*}{ Algorithm } & \multicolumn{3}{c}{ Best solution } & \multirow{2}{*}{ Mean value } \\
\cline { 2 - 4 } & Worst & Median & Best & \\
\hline \multirow{2}{*}{ Genetic Algorithm } & 14.6903 & 13.67437 & 13.61958 & \\
& 14.8136 & 13.65284 & 13.59108 & 14.03211 \\
\multirow{2}{*}{ This paper } & 15.0297 & 13.61673 & 13.60085 & \\
& 13.690736 & 13.990858 & 14.090395 & \\
& 13.590146 & 13.890857 & 13.590850 & 13.85627 \\
\hline
\end{tabular}

Table 3 Results of test function 3

\begin{tabular}{ccccc}
\hline \multirow{2}{*}{ Algorithm } & \multicolumn{3}{c}{ Best solution } & \\
\cline { 2 - 4 } & Worst & Median & Best & \\
\hline \multirow{2}{*}{ Genetic Algorithm value } & 682.8611 & 681.07684 & 680.8007 & \\
& 683.1885 & 681.52563 & 680.6594 & 681.78951 \\
\multirow{2}{*}{ This paper } & 683.8457 & 681.48742 & 680.6603 & \\
& 680.79724 & 681.69040 & 680.68593 & \\
& 681.62065 & 680.65661 & 681.42529 & \\
\hline
\end{tabular}

As shown in Table 1 to Table 3 above, with the proposed methods of outpoint and PSO algorithm combination there are tests for the three test functions, the test results as compared with the use of genetic algorithms, data and results are more stable, and there is no crossover and mutation process in genetic algorithm, easier to achieve. Therefore, integration of PSO algorithm and penalty function improved integration has some value in solving constrained optimization problems, this paper will apply it in Huangshan scenic spot water supply scheduling optimization.

\section{Multi-reservoir Scheduling Optimization Model}

Based on the above experimental results, apply the combination of punish function and particle swarm algorithm to deal with the constraints of Huangshan Mountain water supply scheduling optimization problem, take minimum water shortage rate as the optimization target, this model objective function and constraints are as follows:

The objective function:

$$
\min f=\sum_{i=1}^{W} \sum_{t=1}^{T}\left[\left(Q_{i t}+D_{i t}-G_{i t}\right) / Q_{i t}\right]
$$


Non-negative constraints of water shortage rate:

$\sum_{i=1}^{W} \sum_{t=1}^{T}\left[\left(Q_{i t}+D_{i t}-G_{i t}\right)\right]>0$

Available water supply constraints:

$G_{i t, \min } \leq G_{i t} \leq G_{i t, \max }$

Constraints of water scheduling volume:

$D_{i t, \min } \leq D_{i t} \leq D_{i t, \max }$

In this formula, $f$ refers to the water shortage rate; $\mathrm{W}$ refers to the reservoir number; $\mathrm{T}$ refers to the time span; $Q_{\text {it }}$ means water demand volume of the reservoir i at time $\mathrm{t}$; $G_{\text {it }}$ refers water supply volume of the reservoir $\mathrm{i}$ at time $\mathrm{t} ; D_{\mathrm{it}}$ refers to the water scheduling volume of reservoir $\mathrm{i}$ at time t. Where in, $Q_{\mathrm{it}}$ is calculated by weighted moving average method, the formula can be expressed as:

$$
Q_{\mathrm{it}}=\frac{W_{i} T_{i}+W_{i-1} T_{i-1}+\ldots \ldots+W_{i-n+1} T_{i-n+1}}{W_{i}+W_{i-1}+\ldots \ldots+W_{i-n+1}}
$$

In this formula, $w$ represents the weight of the sliding, $\mathrm{T}$ represents water consumption observation data, $\mathrm{n}$ represents the sliding periods. Using the formula (21), combined with the time span of the obtained water data, daily water consumption can be predictable, weekly or monthly water consumption can be specific to the actual needs.

In this paper, based on 2012 scheduling data, it predicts the Huangshan Scenic hot spring water factory weekly water consumption further optimizes the water, scheduling, data used in the table 4: 
Table 4 weekly water consumtion of Hot Spring water factory (unit: $\left[\mathrm{m}^{3}\right]$ )

\begin{tabular}{|c|c|c|c|}
\hline $\begin{array}{c}\text { Week } \\
1 \sim 12\end{array}$ & $\begin{array}{c}\text { Week } \\
13 \sim 24\end{array}$ & $\begin{array}{c}\text { Week } \\
25 \sim 36\end{array}$ & $\begin{array}{c}\text { Week } \\
37 \sim 48\end{array}$ \\
\hline 4227 & 6279 & 6167 & 7334 \\
\hline 2957 & 5814 & 6702 & 4801 \\
\hline 3388 & 6410 & 6829 & 5883 \\
\hline 5428 & 6384 & 7188 & 5467 \\
\hline 4186 & 5767 & 6986 & 4900 \\
\hline 4576 & 5844 & 6041 & 4205 \\
\hline 4531 & 6238 & 7419 & 4046 \\
\hline 4446 & 6072 & 6836 & 3672 \\
\hline 4463 & 5686 & 5064 & 3147 \\
\hline 4555 & 5866 & 5635 & 3374 \\
\hline 4259 & 5623 & 6364 & 3017 \\
\hline 5133 & 5248 & 5655 & 4374 \\
\hline
\end{tabular}

Simple addition and average on weekly data, monthly water shortage data rate is obtained after optimization, and compared it with Huangshan spot manual scheduling monthly water shortage rate, the results are as follows:

Table 5 comparison chart between manual scheduling with proposed model in this paper

2012 manual scheduling water shortage $(\%)$

proposed model tin this paper water shortage $(\%)$

\begin{tabular}{lll}
\hline Jan. & 11.34 & 8.26 \\
Feb. & 8.41 & 5.97 \\
Mar. & 3.97 & 2.72 \\
Apri. & 4.05 & 3.21 \\
May & 3.76 & 3.04 \\
June & 1.54 & 1.59 \\
July & 0.96 & 0.89 \\
Aug. & 0.28 & 0.64 \\
Sep. & 2.01 & 1.48 \\
Oct. & 4.59 & 3.27 \\
Nov. & 9.34 & 6.21 \\
Dec. & 10.09 & 6.49 \\
\hline
\end{tabular}


As shown in table 5, manual scheduling water shortage rate is 2012 Huangshan Scenic Area's real shortage rate results. Use the methods provided herein to optimize 2012 historical data scheduling, which shows an obvious effect.

The most serious water shortage in Huangshan scenic spot is Nov. Dec., Jan, and Feb. , which water rates have improved significantly. Three months from June to Aug of the wet period, natural rainfall is more abundant and mountain reservoir storage is rich, generally do not have a shortage of water, except for when holidays or great events, that caused a sharp increase in water consumption. So this three months optimization will not be much difference compared with manual scheduling. In short, PSO algorithm and penalty processing algorithm combining in Huangshan Mountain water supply optimization scheduling problem have better results.

\section{Summary and Expectation}

This paper has introduced the PSO algorithm based on reservoir optimization dispatching in Huangshan Scenic, proposed a method of PSO and penalty factor evaluation mechanism to solve nonlinear constrained optimization problems, and use this method of testing to test Huangshan actual water supply scheduling system and the results show that this method is simple, which can solve more reservoir optimal scheduling problems similar to characteristics of Huangshan Scenic spot.

However, this method still exist PSO algorithm easily falling into local optimal solution. According to the scenic mountain-specific environmental and scheduling accuracy requirements, consider using the improved PSO algorithms, such as PSO algorithm based on genetic algorithm, chaos PSO algorithm equal to combining penalty number to solve nonlinear constrained optimization problems. In short, this paper has proposed the method that performs well in Huangshan, if extended to all mountain scenic spot, the algorithm improvement is needed according to the specific application circumstances.

\section{References}

[1] Wang Chunchao, Wang liping, Cao Yunhui etc. application of Improving multivariable grey model in the urban water consumption forecasting $[\mathrm{J}]$. Water and electricity energy science, 2013, $31(2): 27$ to 30 .

[2] Sidhartha Panda,Banaja Mohanty,P.K. Hota. Hybrid BFOA-PSO algorithm for automatic generation control of linear and nonlinear interconnected power systems[J]. Applied Soft Computing Journal, 2013, 1312:1033-1036.

[3] Zhang Jun, cheng, chuntian liao shengli, Zhang Shixin. Application study of Improving particle swarm optimization algorithm in the optimal operation of hydropower stations $[\mathrm{J}]$. Journal of water conservancy, 2009, 435:435-441.

[4] Wang shaobo, Xie Jiancang, Kong Ke. Application of self-Adaptive genetic algorithm in reservoir optimizing [J]. Journal of water conservancy, 2006, 480:480-485. 
[5] JeanBosco Etoa Etoa. A globally convergent sequential linear programming algorithm for mathematical programs with linear complementarity constraints[J]. Journal of Information and Optimization Sciences, 2010, 315: 1011-1019.

[6] Wei, ChihChiang. Exponential smoothing Model Combined with Principal Component Analysis Applied to Quantitative Precipitation Forecast for a Reservoir Watershed during Typhoon Periods[J]. JOURNAL OF HYDROMETEOROLO-GY , 2012, 13(2):722-734.

[7] Ding Genhong Cao Wenxiu. Application of Improved particle swarm algorithm in the reservoir optimizing $[\mathrm{J}]$. Journal of the south-north water diversion and water conservancy science and technology, 2014,01:127-130.

[8] Valipour, Mohammad(Banihabib, Ebrahim M. Comparison of the ARMA, ARIMA, and the autoregressive artificial neural network models in forecasting the monthly inflow of Dez dam reservoir [J]. JOURNAL OF HYDROLOGY, 2013, 476:433-441.

[9] Gan Min Peng Hui. The constrained optimization problem of A new self-adaptive penalty function algorithm [J]. Journal of information and control, 2009,01:24 to 28

[10] Liu Qiongsun, Zhou Shenghua. Hybrid genetic algorithm based on adaptive penalty function method [J]. Journal of chongqing university (natural science edition), 2006 and practices: 78-81.

[11]Mahmud Iwan,R. Akmeliawati,Tarig Faisal,Hayder M.A.A. Al-Assadi. Performance Comparison of Differential Evolution and Particle Swarm Optimization in Constrained Optimization[J]. Procedia Engineering,2012,41:

[12] Liu.Yanmin A hybrid particle swarm optimization (pso) algorithm in solving constrained optimization [J]. Journal of tsinghua university (natural science edition), 2013, 11:242-246. 